\title{
APORTES DE NATALIA KRIVOSHEIN DE CANESE AL ESTUDIO DE LA LENGUA, LA LITERATURA Y LA CULTURA PARAGUAYA
}

\section{Contributions of Natalia Krivoshein de Canese to the study of the language, literature and paraguayan culture}

\author{
Valentina Canese Caballero ${ }^{1}$
}

\section{Resumen}

Natalia Krivoshein de Canese nació en Praga 1926 de padres exiliados rusos quienes emigraron al Paraguay cuando ella tenía 3 años. Comenzó sus estudios de la lengua guaraní en el Instituto de Lingüística Guaraní del Paraguay a los 40 años y al terminarlos y recibir el título de Profesora de Lengua y Cultura Guaraní, continuó en el Instituto Superior de Lenguas de la Universidad Nacional de Asunción donde obtuvo la Licenciatura en Lengua Guaraní. Estando allí, empezó a hacer publicaciones con algunos de sus compañeros y se dedicó al estudio de la realidad lingüística del Paraguay. A través de un enfoque cualitativo descriptivo, este artículo tiene como objetivo principal describir la trayectoria académica y el aporte realizado por Natalia Krivoshein de Canese al estudio y la promoción de la lengua y la cultura guaraní. Algunos de sus aportes más importantes incluyeron su obra -Gramática de la Lengua Guaraníl, el diccionario guaraní-español, españolguaraní "Ñe 'ẽ ryru" y la Revista Bilingüe Nemity fundada con Feliciano Acosta y Tadeo Zarratea. Con estos autores también publicó varias recopilaciones y traducciones de cuentos y fábulas en formato bilingüe. El libro -El español del Paraguay: en contacto con el guaraníl publicado en coautoría con Graziella Corvalán es también de importante referencia. Muchas de sus obras fueron reeditadas, algunas múltiples veces como es el caso del diccionario "Ñe "ẽ ryru". Además, publicó numerosos artículos en revistas académicas. Durante varios años ejerció la docencia universitaria en el Instituto Superior de Lenguas de la UNA donde se dedicó a formar a futuros estudiosos de la lengua guaraní. A través de este análisis, se puede concluir que, gracias a su dedicación al estudio y la promoción de la lengua y la cultura guaraní, Natalia Krivoshein se convirtió en un referente nacional e internacional en su área. Así, su invaluable legado continúa vigente a través de su obra y de todos aquéllos que fueron sus estudiantes y compañeros en el estudio de la lengua guaraní.

${ }^{1}$ PhD Curriculum e Instrucción, Directora del Instituto Superior de Lenguas, Facultad de Filosofía, Universidad Nacional de Asunción. vcanese@fil.una.py

$\tilde{N} E M I T \bar{Y} R \tilde{A}, 2019 ; 1(1)$

DOI: https://doi.org/10.47133/nemityra010102 
Palabras clave: Natalia Krivoshein de Canese, lingüista, lengua guaraní, bilingüismo paraguayo.

\section{Abstract}

Natalia Krivoshein de Canese was born in Prague 1926 from Russian exiled parents who emigrated to Paraguay when she was 3 years old. She began her studies of the Guaraní language at the Paraguay Institute of Guaraní Linguistics at age 40 and upon finishing them and receiving the degree of Guaraní Language and Culture teacher, she continued at the National University of Asunción's Higher Institute of Languages where she obtained a Bachelor's degree in Guaraní language. While there, she began publishing with some of her colleagues and devoted herself to the study of the linguistic reality in Paraguay. Through a descriptive qualitative approach, this article 's main purpose is to describe the academic trajectory and contribution made by Natalia Krivoshein de Canese to the study and promotion of the Guaraní language and culture. Some of her most important contributions include her works: "Grammar of the Guaraní Language", the Guaraní-Spanish, Spanish-Guaraní "Ñe "ẽ ryru" dictionary and the Bilingual Magazine - Nemityl founded with Feliciano Acosta and Tadeo Zarratea. With these authors she also published several compilations and translations of stories and fables in bilingual format. The book "The Spanish of Paraguay: in contact with Guarani" published in co-authorship with Graziella Corvalán is also an important reference. Many of her works have been reprinted, several times as is the case with the "Ñe "ẽ ryru" dictionary. She also published numerous articles in academic journals. For several years she taught at the Higher Institute of Languages of the UNA where he dedicated herself to training future students of the Guaraní language. Through this analysis, it can be concluded that through her dedication to the study and promotion of the Guaraní language and culture, Natalia Krivoshein became a national and international reference in her field. Thus, her invaluable legacy continues through her work and her students and colleagues who study the Guaraní language.

Keywords: Natalia Krivoshein de Canese, linguist, guaraní language, paraguayan bilingualism

\section{Introducción}

La lengua y la cultura guaraní constituyen elementos fundamentales de la identidad del Paraguay ya que es uno de los pocos países donde una lengua de origen 


\section{ISSN 2707-1642}

indígena permaneció como lengua de comunicación principal de la población luego de siglos de colonización española (Krivoshein de Canese, 1997b). Como tal, ha sido el enfoque de estudio de numerosos académicos a través de la historia entre los que se encuentra Natalia Krivoshein de Canese, quien durante más de tres décadas se convirtió en uno de los principales referentes nacionales en el estudio y la promoción de la lengua y la cultura guaraní. De origen ruso, nació en Praga 1926 de padres exiliados quienes emigraron al Paraguay cuando ella tenía 3 años. Su padre, el Dr. Nicolás Krivoshein fue invitado por el gobierno paraguayo para juntamente con otros ingenieros rusos exiliados formar parte de la fundación de la Facultad de Ciencias Físicas y Matemáticas donde se desempeñó como docente por varios años para luego mudarse a la Argentina y participar de la fundación de la Facultad de Ingeniería en la Universidad de la Plata.

Según los recuerdos de Natalia, a pesar de ya saber leer y escribir en ruso, cuando inició sus estudios primarios tuvo dificultades al principio pues no comprendía el español que la profesora hablaba. Fue su padre, que tenía conocimientos del español quien la acompañó en estos inicios y quizás por este motivo desarrolló una empatía especial para con los paraguayos que no recibían la educación en su lengua materna. Los estudios primarios los realizó primeramente en la Escuela Antequera y luego en el Colegio Italiano. Posteriormente, ingresó al Colegio Internacional donde culminó sus estudios secundarios.

Inició los estudios de ingeniería de la Universidad Nacional de Asunción, pero no los pudo culminar porque decidió dedicarse completamente a la crianza de sus hijos y recién a los 40 años tuvo la oportunidad de volver a estudiar, esta vez la lengua guaraní. Ella manifestó en varias entrevistas (ABC, 2005) que siempre le había interesado esta lengua debido al arraigo que tiene con nuestro país que ella adoptó como suyo. Inició sus estudios de esta lengua en el Instituto de Lingüística Guaraní del Paraguay. Al terminarlos y recibir el título de Profesora de Lengua y Cultura Guaraní, continuó en el Instituto Superior de Lenguas de la Universidad nacional de Asunción donde obtuvo la Licenciatura en Lengua Guaraní.

Ya como estudiante, empezó a hacer publicaciones con algunos de sus compañeros y se dedicó al estudio de la realidad lingüística del Paraguay. De esta manera fue convirtiéndose en una de las grandes promotoras y referentes de la lengua guaraní durante el transcurso de su carrera. Este artículo tiene como objetivo principal describir la trayectoria académica y el aporte realizado por Natalia Krivoshein de Canese al estudio y la promoción de la lengua y la cultura guaraní. 


\section{Metodología}

La metodología utilizada tuvo un enfoque cualitativo y alcance descriptivo en donde se utilizaron datos provenientes de las publicaciones realizadas por la autora, así como entrevistas e historias de vida para llegar a una descripción e interpretación de la perspectiva de los autores sobre los eventos y aportes más importantes en la carrera de la autora objeto de estudio (Chárriez Cordero, 2012; Ruiz Olabuénaga, 2012).

El estudio fue delimitado temporalmente al período de 1977 a 2007, que corresponde al periodo en que la autora estuvo activa publicando trabajos de investigación y otras obras referentes a la lengua guaraní. Las técnicas empleadas fueron: análisis de información, entrevistas, y la historia de vida de la autora objeto de estudio. A través del análisis de información se pueden captar, evaluar, seleccionar y sintetizar los mensajes de un texto, mediante el estudio contextualizado de sus significados, con relación a un problema determinado. En este estudio, se utilizaron estrategias de búsqueda y análisis que permitieron tener un panorama amplio del trabajo realizado por la autora (Dulzaides Iglesias \& Molina Gómez, 2004). Se registraron y analizaron las publicaciones y referencias sobre la autora objeto de estudio disponibles en los buscadores y repositorios disponibles en bibliotecas online a través de Google Académico, el Portal Guaraní, y otros.

Además, se utilizaron registros de entrevistas realizadas con la autora objeto del estudio donde la misma narra su perspectiva con respecto al guaraní y su importancia en la sociedad paraguaya. Una de ellas publicada en el diario ABC Color (2005) y la otra entrevista inédita realizada por la autora de este artículo el 20 de diciembre de 2005 como parte de un estudio preliminar piloto para su estudio de doctorado en la que se utilizaron métodos fenomenológicos de entrevista cualitativa siguiendo las recomendaciones de Seidman (2006). A partir de estos datos se pudo realizar el análisis sobre el aporte de la obra de Natalia Krivoshein al estudio de la lengua guaraní en Paraguay.

\section{Resultados}

De acuerdo a la entrevista publicada por el diario ABC (2005), Natalia Krivoshein, a pesar de ser multilingüe pues hablaba el ruso y el español, además del inglés y el francés, quiso estudiar el guaranì -porque es el idioma que todo el mundo habla en este país. Las canciones que escuchaba y las comedias que iba a ver al teatro no entendía. Tampoco asimilaba lo que escuchaba en la radio o en la calle. Por eso 


\section{ISSN 2707-1642}

quería aprenderl (s.p.). En el momento de la entrevista también indicó que -sigo aprendiéndolo porque no es mi primera lenguall (s.p.).

Además, cuenta que, al estudiar guaraní, lo que más le interesó fue la gramática y los estudios gramaticales ya que eso siempre le gustó. Por eso, recuerda, podía ayudar a sus compañeros y colegas en esta área. Así fue que juntamente con Feliciano Acosta y Tadeo Zarratea fundaron la Revista Ñemity en el año 1977 donde decidieron publicar artículos y otros materiales sobre la lengua guaraní y el bilingüismo paraguayo. Esta revista bilingüe respondió a la necesidad de publicar materiales académicos y culturales referentes a la lengua, la lingüística y la cultura guaraní. Se publicaron un total de 44 números hasta el año 2002. Colaboraron en esta publicación muchos de los referentes académicos y culturales de la lengua guaraní tales como Shaw Gynan (1998) y Bartolomeu Meliá (2001) entre otros.

Además, con su esposo el Dr. Arquímedes Canese y el Prof. Almidio Aquino, publicaron - Nociones de Guaraní para Uso Médicol $(1977,1980,1998)$. El propósito de este material es el de servir de referencia para -los doctores [que] son de clase alta y casi no hablan guaraní. Cuando practican en el Hospital de Clínicas no pueden comunicarse con un enfermo que viene del interior. Incluye diálogos del médico con el enfermo, un diccionario de palabras médicas y algunas palabras necesariasl. Así, ella agrega, — con estos aportes, nuestro idioma goza de buena salud. \| (Abc, 2005: s.p.)

En 1983 publica por primera vez su -Gramática de la Lengua Guaraníl que sigue siendo un referente para muchos estudiosos de la lengua guaraní. Este material fue reeditado varias veces (1998), luego ya en coautoría con Feliciano Acosta como -Gramática Guaraníl (2001). Teniendo en cuenta ambas publicaciones es su segunda obra más citada según el registro del Google Académico. Esta obra ha sido citada ampliamente por varios autores y en múltiples idiomas (Nordhoff, S., 2004; Mello-Wolter, R. M. ,2007; Dude, S., 2008; Dietrich, W., 2010 2017;

Lustig, 2010; Frutos, L., 2011; Estigarribia, 2017; Zubizarreta, 2017) ya que presenta un análisis bastante completo de la gramática guaraní.

El libro -El español del Paraguay: en contacto con el guaraníl (1987) que publica en coautoría con Graziella Corvalán es su obra más referenciada según los registros de Google Académico. Este libro sigue siendo de referencia para los académicos que estudian la situación de contacto lingüístico que existe en nuestro país. Estudiosos de la lengua española tanto paraguayos como a nivel internacional referencian esta obra ya que fue una de las primeras en analizar al castellano paraguayo desde el punto de vista linguiístico en contacto con el guaraní (De Granda, 1996; Alcaine, 1997; Lipski, 1998, 2007; Choi, 2000, 2001; Rendón, 


\section{ISSN 2707-1642}

2008; Lustig, 2010; Velázquez-Castillo, M. \& Henderson, M. H., 2013; Mortimer, 2016; Estigarribia, B. 2017). Así, este trabajo continúa siendo de utilidad para los lingüistas interesados en la situación de contacto lingüístico entre el guaraní y el español en Paraguay.

Conjuntamente con un grupo significativo de estudiosos y defensores de la lengua guaraní, fue una de las principales impulsoras de la Educación Bilingüe en Paraguay, propuesta que se hizo realidad a partir de la Constitución Nacional de 1992 y de la reforma educativa de 1994 (Canese Caballero, 2008). Históricamente, hasta ese entonces, sin importar la lengua materna de los niños, la enseñanza se realizaba en español, lo cual excluía y dificultaba el aprendizaje de los niños de habla materna guaraní. Por este motivo, y como fue mencionado más arriba, posiblemente por sus antecedentes como alumna escolarizada en una lengua no materna, elaboró un proyecto denominado - La Educación Bilingüe para el Paraguay\| (1986), publicado en Estudios Paraguayos Vol. XXI, No. 2, que tuvo un importante lugar en la discusión de ideas y propuestas de cambio educativo.

Con Feliciano Acosta publica múltiples obras, incluyendo la más difundida: "Ñe 'ẽ ryru", diccionario guaraní-español, español-guaraní (1990). También publicaron recopilaciones y traducciones de cuentos y fábulas en formato bilingüe: $\mathrm{Ka}$ ' $\mathrm{i}$ Rekovekue (1994), Ka'i rembiasakue (1994), Tetãgua remimombe'u (1996), Mombe 'ugua 'u (1999) y Ñe 'ẽpoty Aty (2005), así como el material educativo -Jaleéna Guaranìmel (1992). Con Tadeo Zarratea publica la obra "Morangu, Fábulas y Cuentos Populares Universales" (2004). Estos libros los publicó con el afán de brindar a educadores de materiales significativos para la enseñanza de la lengua guaraní como lo fue el material didáctico -Ñembosaraill (1997). Muchas de estas obras fueron reeditadas, algunas múltiples veces como es el caso del diccionario "Ñe "ẽ ryru".

Además, publicó numerosos artículos, entre ellos: "Influencia del bilingüismo en la literatura paraguayall, Ñemity (Asunción) 5 (1980): 10-12; Krivoshein de Canese, Natalia. "Evolución del bilingüismo paraguayo." En: Ñemity 31 (1995): 13-14;

-Sobre la unificación del alfabeto de la lengua guaraní." Suplemento Antropológico 21.2 (1986): 141-179; -El guaraní como lengua aglutinante y polisintética." Suplemento Antropológico (1995): 1-2; y, "Cultura y bilingüismo en el Paraguay." (2001). Publicó regularmente artículos en la Revista Ñemity desde su inicio hasta su último número en 2002. Estos artículos sirvieron y siguen sirviendo de referencia a estudiosos de la lengua guaraní. Durante varios años ejerció la docencia universitaria en el Instituto Superior de Lenguas de la UNA donde se dedicó a formar a futuros estudiosos de la lengua guaraní. 


\section{Conclusión}

Analizando la trayectoria de Natalia Krivoshein de Canese, se puede concluir que con su aporte contribuyó a -la buena salud del guaraníl como lo había expresado en una de las entrevistas. A través de su dedicación al estudio y la promoción de la lengua y la cultura guaraní, se convirtió en un referente nacional e internacional en el estudio de esta lengua. Sirvió de inspiración a muchas personas, inculcando la importancia y el valor de nuestra lengua, especialmente en el ámbito educativo. Su trabajo desinteresado para la promoción y el desarrollo de la lengua guaraní dejó un legado innegable que continúa vigente a través de su obra y de todos aquéllos que fueron sus estudiantes y compañeros. A partir de este aporte, la comunidad académica debe seguir apuntando a que cada día se realicen más estudios sobre la lengua guaraní, así como sobre la realidad sociolingüística y educativa de nuestro país. Todavía queda mucho por hacer y debemos seguir el ejemplo de Natalia Krivoshein y cada uno de nosotros aportar nuestro grano de arena en esta incansable labor.

\section{Referencias}

Abc. (2005, 13 de marzo). La gringa guaraní. Recuperado de https://www.abc.com.py/edicion-impresa/suplementos/abc-revista/lagringa-guarani-817804.html.

Acosta Alcaraz, F., \& Krivoshein de Canese, N. (1994). Ka 'i rekovekue-La vida de Ca 'i. Traducción al castellano de Natalia Krivoshein de Canese, Asunción: RP ediciones.

Acosta, F., \& de Canese, N. K. (2003). Mombe 'ugua 'u: colección de mitos, fábulas y leyendas paraguayas. Asunción: Servilibro.

Acosta Alcaraz, F., \& Zarratea, T. (2003). Ka 'i rembiasakue (Las aventuras de ca ‘i). Traducción al español por Natalia Krivoshein de Canese. Asunción: Servilibro.

Acosta Alcaraz, F. \& Krivoshein de Canese, N. (2005). Ne ‘ẽpoty Aty - Voces de Poetas en guaraní. Asunción-Paraguay: Editorial Servilibro.

Alcaine, A. P. (1997). Acerca del contacto de lenguas español y guaraní. In Actas del I Simposio Internacional sobre Bilingüismo: Comunidades $e$ Individuos Bilingües (pp. 807-817).

Canese Caballero, V. (2008). When policy becomes practice: Teachers' perspectives on official bilingualism and the teaching of Guarani as a second language in Paraguay. Arizona State University.

Chárriez Cordero, M. (2012). Historias de vida: Una metodología de investigación cualitativa. Revista Griot, 5(1), 50-67. Año 2012- Puerto Rico. Recuperado de https://revistas.upr.edu/index.php/griot/article/view/1775. 
Choi, J. K. (2000). Direct Object Drop: The Genetic Cause of a Synthactic Feature in Paraguayan Spanish. Hispania, 531-543.

Choi, J. K. (2001). The genesis of voy en el mercado: The preposition en with directional verbs in Paraguayan Spanish. Word 52, no. 2: 181-196.

Corvalán, G., Krivoshein de Canese, N., Hobbs, C. M. and Rivarola, D. (1993). Educación bilingüe. In Harvard Institute for International Development and Centro Paraguayo de Estudios Sociológicos, Análisis del Sistema Educativo en el Paraguay. Sugerencias de Política y Estrategia para su Reforma. 357-374. Asunción, Paraguay: Centro Paraguayo de Estudios Sociológicos.

De Granda, G. (1996). Interferencia y convergencia sintácticas e isogramatismo amplio en el español paraguayo. International Journal of the Sociology of Language, 117(1), 63-80.

Dietrich, W. (2010). Problems of Distinguishing Nominal Compounding from Syntactic and Noun Categorization Devices in Tupi-Guarani Languages. In Linguistics and Archaeology in the Americas (pp. 11-26). BRILL.

Dietrich, W. (2010). Sintaxis del guaraní chaqueño (chiriguano, tupí-guaraní): la cláusula y las relaciones interclausales. Amerindia, 33(34), 333-363.

Dietrich, Wolf. "Word classes and word class switching in Guaraní Syntax." In Guarani linguistics in the 21st century, pp. 158-193. Brill, 2017.

Drude, S. (2008). Die Personenpräfixe des Guaraní und ihre lexikographische Behandlung. In Geschichte und Aktualität der deutschsprachigen Guarani-Philologie: Akten der Guaraní-Tagung in Kiel und Berlin 25.27. Mai 2000 (pp. 198-234). Lit Verlag.

Estigarribia, B. (2015). Guarani-Spanish Jopara Mixing in a Paraguayan Novel: Does it Reflect a Third Language, a Language Variety, or True Codeswitching. Journal of Language Contact, 8(2), 183-222.

Estigarribia, B. (2017). A grammar Sketch of Paraguayan Guarani. In Guarani linguistics in the 21st century (pp. 7-85). BRILL.

Estigarribia, B. (2017b). Guarani Morphology in Paraguayan Spanish: Insights from Code-Mixing Typology. Hispania, 100(1), 47-64.

Estigarribia, B. (2017c). Insertion and Backflagging as Mixing Strategies Underlying Guarani-Spanish Mixed Words. In Guarani Linguistics in the 21st Century (pp. 315-347). Brill.

Frutos, L. (2011). Escalas no Guaraní Paraguaio: uma análise do modificador de grau-pa (Doctoral dissertation, Universidade de São Paulo).

Gynan, S. (1998). El Futuro del Bilingüismo paraguayo. Nemity: Revista Bilingüe de Cultura, $\mathrm{N}^{\circ} 36,23-25$.

Krivoshein de Canese, N., Arquímedes, C. \& Aquino, A. (1977). Nociones de guaraní para uso médico-Revista Paraguaya de microbiología 12: $\mathrm{N}^{\circ} 1$ pág. 13, 14. 
Krivoshein de Canese, N. (1980). Natalia, - - Influencia del bilingüismo en la literatura paraguayall, Ñemity (Asunción), (5), 10-12.

Krivoshein de Canese, N., Canese, A., \& Aquino, A. (1980). Nociones de idioma guaraní para uso médico. Facultad de Ciencias Médicas, Cátedra de Bacteriología y Parasitología, Universidad Nacional de Asunción.

Krivoshein de Canese, Natalia. 1983. Gramática de la lengua guaraní. Asunción, Paraguay: Colección Nemity.

Krivoshein de Canese, N. (1986). Sobre la unificación del alfabeto de la lengua guaraní. Suplemento antropológico, (21), 141-179

Krivoshein de Canese, N., \& Corvalán, G. (1987). El español del Paraguay en contacto con el guaraní. Asunción, Centro Paraguayo de Estudios Sociológicos.

Krivoshein de Canese, N. (1989). Educación bilingüe para el Paraguay.

Documento de Trabajo $\mathrm{N}^{\circ}, 16$.

Krivoshein de Canese, N., \& Acosta Alcaraz, F. (1990). Ñe'eryru-Diccionario guaraní-español. Asunción, Paraguay: Colección Ñemity.

Krivoshein de Canese, N., Zarratea, T. \& Acosta Alcaraz, F. (1992). Jaleéna Guaraníme. Asunción, Paraguay: Base Ecta.

Krivoshein de Canese, N. (1993). Variedad de guaraní que se usaría en Ia educación. Nemity. Asunción, (26), 14-18.

Krivoshein de Canese, N. (1993b). Cultura y bilingüismo en el Paraguay. Suplemento antropológico, (28), 107-120.

Krivoshein de Canese, Natalia. 1994. Gramática de la lengua guaraní. Asunción, Paraguay: Colección Ñemity.

Krivoshein de Canese, N. (1995). El guaraní como lengua aglutinante y polisintética. En: Nemity, 31, 7-12.

Krivoshein de Canese, N. (1995b). Evolución del bilingüismo paraguayo. En:

Nemity, 31, 13-14.

Krivoshein de Canese, N. (1996). Apuntes de lingüística general y aplicada.

Asunción: la autora.

Krivoshein de Canese, N. (1997). El guaraní como lengua aglutinante y

polisintética. Suplemento antropológico, (32), 317-328.

Krivoshein de Canese, N. (1997b). Cultura y bilingüismo en el Paraguay, en

Suplemento Antropológico, vol. 32, núm. 1-2, pp. 111-112.

Krivoshein de Canese, N. (1997). Nembosarái: guaraní como segunda lengua. Asunción: la autora.

Krivoshein de Canese, N. y Acosta Alcaraz, F. (1997). Ñe ‘ẽryru avaðe 'ẽ - karaiðe 'ẽ, karaiðe 'ẽ - avaðe 'ẽ, Diccionario guaraní-español, español- guaraní. Asunción: Universidad Nacional de Asunción (Colección Ñemitỹ).

Krivoshein de Canese, Natalia. 1998. Gramática de la lengua guaraní. Asunción: Colección Ñemitỹ. 
Krivoshein de Canese, N., Canese, A., \& Aquino, A. (1998). Nociones de idioma guaraní para uso médico. Facultad de Ciencias Médicas, Cátedra de Bacteriología y Parasitología, Universidad Nacional de Asunción.

Krivoshein de Canese, Natalia. 1999. La polisíntesis (Gramática guaraní III). Nemity, 37, 7-12.

Krivoshein de Canese, N., \& Alcaraz, F. A. (2001). Gramática guaraní. Colección Ñemitỹ, Asunción.

Krivoshein de Canese, N., \& Alcaraz, F. A. (2002). Nemombe'u guaraní Jeporavo, Colección de Cuentos en guaraní. Colección Ñemitỹ, Asunción.

Krivoshein de Canese, N. \& Zarratea, T. (2004). Morangu: fábulas y cuentos populares universales. Asunción, Paraguay: ServiLibro

Krivoshein de Canese, N., Gamba, C. M., \& Alcaraz, F. A. (2005). Tetãgua remimombe‘ $u$ : Cuentos populares paraguayos. Asunción, Paraguay: Servilibro.

Krivoshein de Canese, N., \& Alcaraz, F. A. (2007). Ñemombe'u guaraní Jeporavo, Colección de Cuentos en guaraní. Colección Ñemitỹ, Asunción.

Lipski, J. M. (1998). Spanish Linguistics: The Past 100 Years: Retrospective and Bibliography. Hispania, 248-260.

Lipski, J. M. (2007). El español de América en contacto con otras lenguas.

Lingüística aplicada del español, 309-345.

Lustig, W. (2010). Mba'éichapa oiko la guarani? Guaraní y jopara en el Paraguay. PAPIA-Revista Brasileira de Estudos do Contato Linguístico, 4(2), 19-43.

Melià, B. (2001). El Guaraní Popular y el Guaraní Impopular. Nemity: Revista Bilingüe de Cultura, $\mathrm{N}^{\circ} 41,14-16$.

Mortimer, K. S. (2016). Producing change and stability: A scalar analysis of Paraguayan bilingual education policy implementation. Linguistics and Education, 34, 58-69.

Nordhoff, S. (2004). Stative verbs and possession in Guaraní. In Syntax of the Worlds Languages" Conference, Leipzig.

Olabuénaga, J. I. R. (2012). Metodología de la investigación cualitativa (Vol. 15). Universidad de Deusto.

Mello-Wolter, R. M. (2007). El léxico del parentesco en el guaraní paraguayo ¿un yopará? Signos Lingüísticos, 3(06).

Rendón, J. G. (2008). Typological and social constraints on language contact: Amerindian languages in contact with Spanish. Netherlands Graduate School of Linguistics.

Seidman, I. (2006). Interviewing as qualitative research: A guide for researchers in education and the social sciences. Teachers college press.

Velázquez-Castillo, M., \& Henderson, M. H. (2013). Quiero para mi novio: Aspectual uses of para in Paraguayan Spanish. Journal of Pidgin and Creole Languages, 28(1), 65-102. 
ISSN 2707-1642

Zubizarreta, M. L., \& Pancheva, R. (2017). A formal characterization of personbased alignment. Natural Language \& Linguistic Theory, 35(4), 1161-120 